
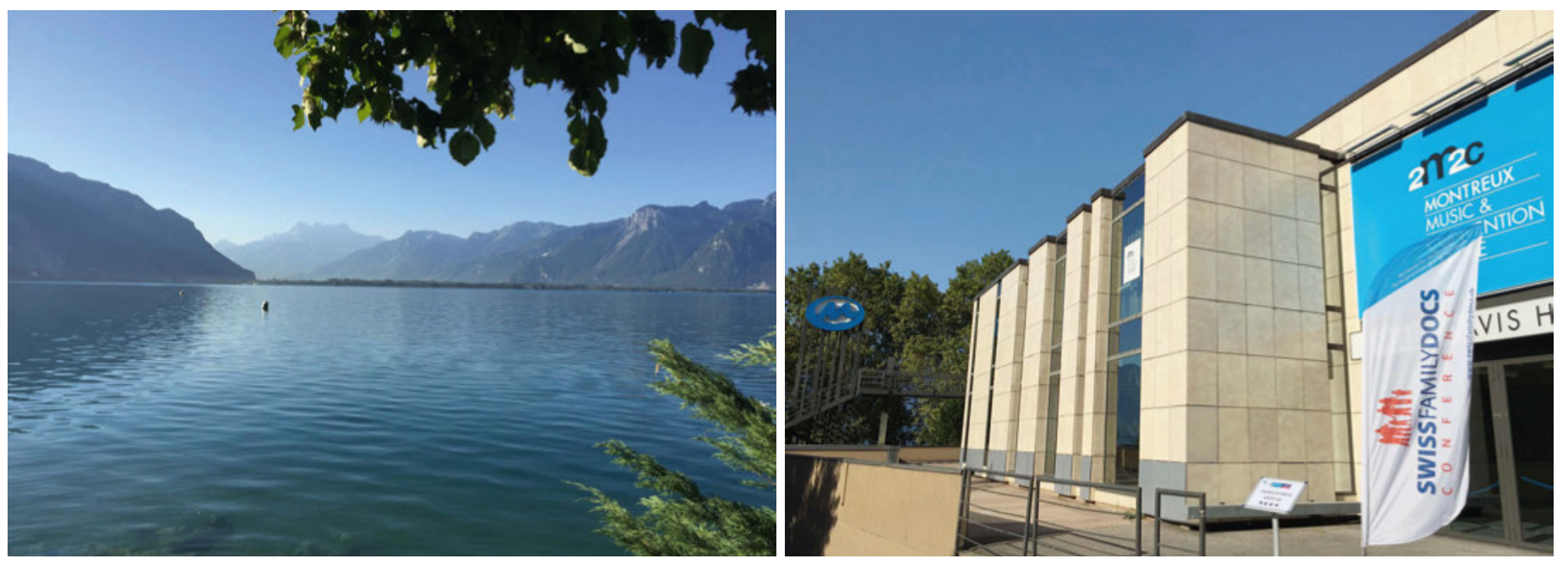

Swiss Family Docs Conference vom 8. und 9. September 2016 in Montreux

\title{
Weniger Eigensinn, mehr Teamarbeit
}

\section{Felicitas Witte}

Dr. med., Ärztin und Journalistin

Arbeitet man im Team, verbessert das das Outcome - und es macht mehr Spass. «Zusammen» hiess das Motto der diesjährigen Swiss Family Docs Conference, die Anfang September in Montreux stattfand. Wie gute Zusammenarbeit funktioniert und welche Kompetenzen man dafür braucht, war eines der zentralen Themen am Kongress.

Es waren nicht die besten Voraussetzungen für die Referenten an der Swiss Family Docs Conference. Wie soll man die Kollegen für seinen Vortrag faszinieren, wenn draussen die Sonne von einem wolkenlosen Himmel scheint? Doch trotzdem waren Seminare und Workshops rege besucht, und auch in den Pausen am Ufer des Genfersees hörte man die Teilnehmer angeregt über die Vorträge diskutieren.

Den Organisatoren lag ein Thema besonders am Herzen, was sie zum Motto des Kongresses machten: «Zusammen». Eigentlich tönt es logisch: Nur wenn man fruchtbar und effektiv zusammen arbeitet, Stärken bündelt und Synergien schafft, kommt das Beste für den Patienten heraus. «Unser Gesundheitssystem ist aber bisher nicht organisiert, um in dieser Art zu praktizieren", sagte Marc Müller, langjähriger Präsident von "Hausärztinnen und -ärzte Schweiz» und Allgemeinarzt in Grindelwald. "Wir müssen die Gesundheitspolitiker überzeugen, wie wichtig interprofessionelles Zusammenarbeiten ist, und dass entsprechende Strukturen dafür geschaffen werden müssen.»

Die Art, in der junge Ärztinnen und Ärzte heute Medizin praktizieren würden, habe sich geändert, sagte Ivy Oandasan von der Universität in Toronto, vor allem im Hausarztbereich. «Die neue Millennium-Generation möchte mehr Work-Life-Balance. So braucht man heute vielleicht zwei Hausärzte, um den früheren Posten von einem zu besetzen.» Die neue Generation möchte im Team arbeiten. «Wir von der älteren Generation kennen das nicht so, aber die Jüngeren sind daran gewöhnt. Darauf müssen wir uns einstellen.» Zehn Minuten hätten Hausärzte im Schnitt, um einen 


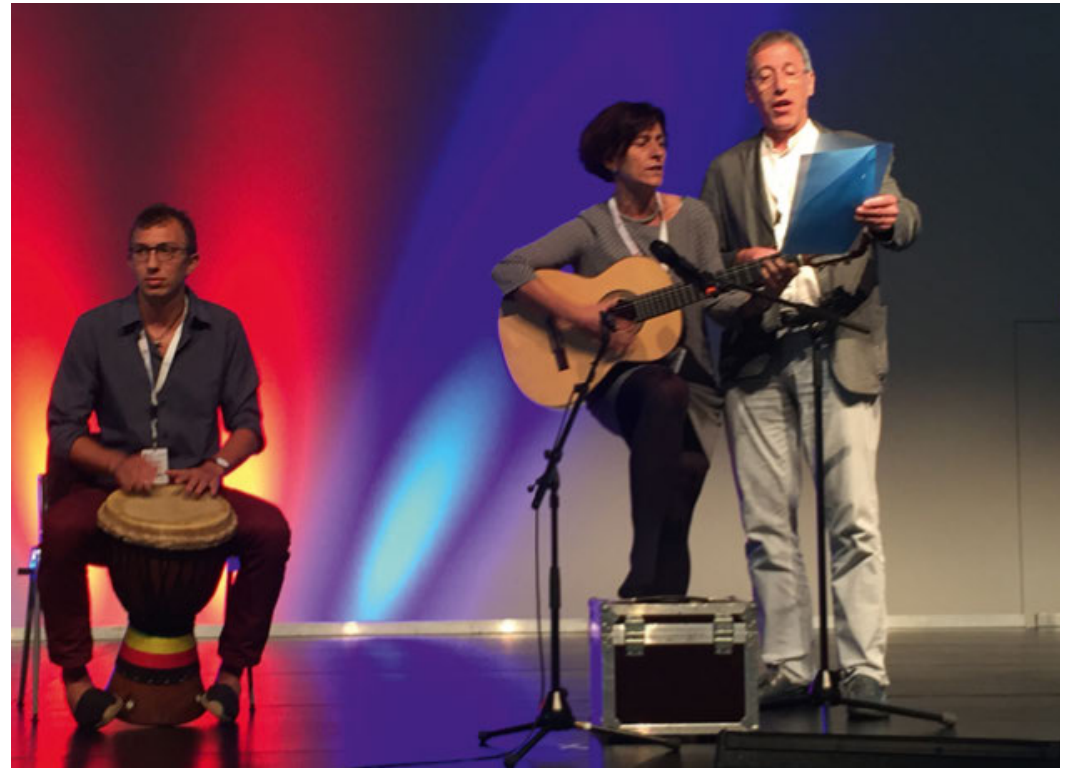

Zu den Klängen des bekannten Chansons Aux Champs-Elysées von Joe Dassin sangen die Organisatoren eine mitreissende Einführung und machten Lust auf den Kongress. Il y a tout ce que vous voulez, lautete der umgedichtete Refrain, und so war es auch: Ein Kaleidoskop spannender Themen wurde präsentiert.

Patienten zu sehen. «Das reicht hinten und vorne nicht.» Menschen leben länger und leiden viel öfter unter chronischen Krankheiten - das braucht Zeit für Gespräche und Erklärungen, damit der Patient alles gut versteht. Ob es die richtige Lösung sei, mehr Ärzte auszubilden, bezweifelt Oandasan. «Wir wissen ja nicht, wo die Ärzte dann hingehen. Vielleicht ergreifen viele von denen nichtmedizinische Berufe.»

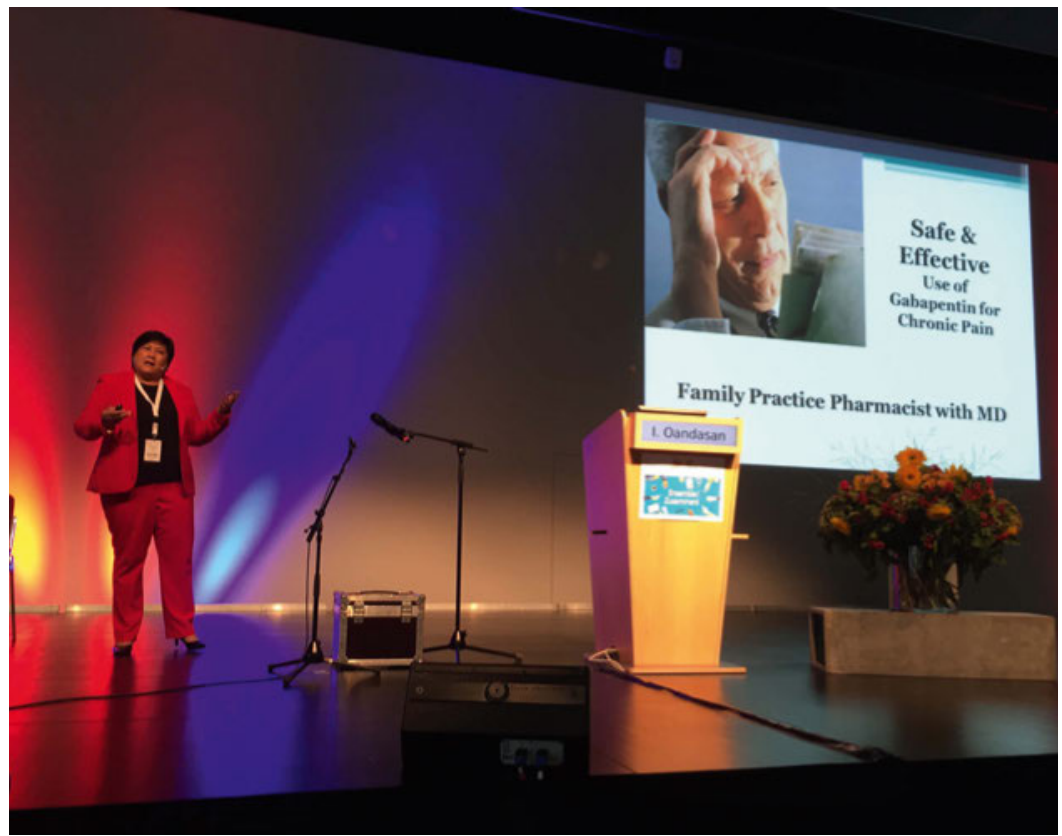

«Wir müssen interprofessionell zusammenarbeiten. Nicht nur weil es nett ist, sondern weil es die Qualität der Behandlung verbessert. Ein Hindernis ist aber oft der Charakter der Ärzte.»

\section{Charakter der Ärzte ist eine Hürde}

Fühlt sich der Patient beim Hausarzt und seinem Team wie in einem «Zuhause» aufgehoben, geht es ihm gesundheitlich besser und man spart auch noch Kosten, wie Wissenschaftler aus Boston 2004 zeigten [1]. «Wir müssen zusammen arbeiten», sagte Oandasan. «Nicht nur, weil es nett ist, sondern weil es die Qualität der Behandlung verbessert.» Und das sei auch ein schlagendes Argument für die Gesundheitspolitiker. Inzwischen gäbe es einige Belege, berichtete Oandasan, dass interprofessionelles Arbeiten das Outcome verbessere, zum Beispiel von Menschen mit chronischen Krankheiten, beim Thema Impfungen oder bei der Aufklärung von sexuell übertragbaren Krankheiten.

Wie das aussehen kann, zeigte die Professorin anhand ihrer Patienten: Barry, ein junger Mann mit Typ-1-Diabetes, hatte lange Zeit seine Zuckerwerte nicht im Griff. Dann hielt eine Pflegerin der Allgemeinarztpraxis Rücksprache mit Hausarzt und Endokrinologen und erklärte Barry ausführlich, worauf er achten muss. Damit besserten sich seine Zuckerwerte deutlich. Bei Allison, einer jungen, alkoholabhängigen Frau, die sich nicht mehr um ihre Kinder kümmern konnte, nahm die Professorin Kontakt zu Sozialarbeiter, Abhängigkeitsberater und Kinderarzt auf, die Allison bei ihrem Alkoholproblem und bei der Versorgung ihrer Kinder unterstützten. Eine Teenager-Mutter mit einem extrem übergewichtigen Baby brachte Oandasan mit einer Diätberaterin und einer Pflegerin in Kontakt und die junge Frau wusste nach ausführlichen Erklärungen, wie sie ihr Kind füttern sollte.

Ein Hindernis an der interprofessionellen Zusammenarbeit sei oft der Charakter der Ärzte. «Viele denken, nur sie selbst könnten bestimmte Untersuchungen machen, und trauen das Nicht-Medizinern nicht zu.» Das sei wie früher im Sandkasten. «Dort will man auch nicht, dass andere Kinder mitspielen. Aber wir müssen interprofessionelles Arbeiten lernen - und zwar von Anfang an.» In Kanada sei es inzwischen in manchen Fächern Pflicht, dass Medizinstudierende und Auszubildende von Gesundheitsberufen miteinander lernen Ein «gut funktionierender interprofessionell arbeitender Sandkasten» brauche jedoch nicht nur Teamwork, sondern auch Forschung, Führung und Finanzierung. «Dafür müssen sich Ärzte einsetzen.»

\section{So geht erfolgreiches Arbeiten im Team}

Wie sich interprofessionelles Arbeiten erfolgreich in die Praxis umsetzen lässt, berichteten Marc Jungi und Nicole Joos. Jungi ist Leitender Arzt der Sanacare Gruppenpraxis in Bern und Joos dort Medizinische 


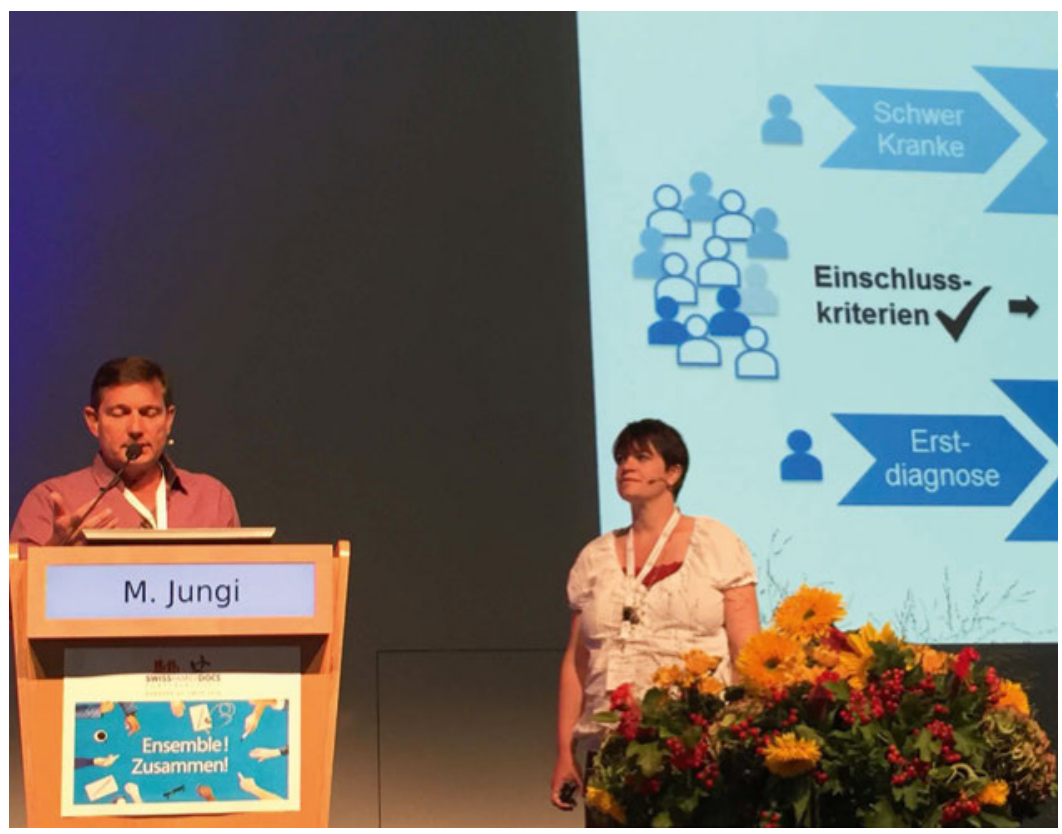

Marc Jungi, Leitender Arzt der Sanacare Gruppenpraxis in Bern: «Wer gute Ideen hat, sollte sich nicht durch noch nicht ausgereifte Rahmenbedingungen abschrecken lassen.» aus Arzt und Coach evaluiert die Krankengeschichte, untersucht den Patienten, stellt die Diagnose und vereinbart Ziele (Abb. 4). Der Patient sieht im Rahmen seines strukturierten Behandlungspfades seinen Coach und seinen Arzt und natürlich jederzeit im Notfall ebenfalls einen Arzt. Bei Problemen überweist dieser zum Spezialisten, der ebenfalls Teil des CCM-Teams ist. «Das sieht starr aus, aber wir passen es individuell an den Patienten an", sagte Jungi. Für Hausarzt und Coach gibt es Leitlinien, Algorithmen und Checklisten, und für die Patienten Broschüren, Notfallkarten und andere Hilfsmittel. Alle Dokumentationen sind in der elektronischen Krankenakte gespeichert oder online verfügbar.

\section{Genau aufgeschrieben, wer was darf}

Nicole Joos mag ihre neue Aufgabe als Coach sehr. Sie schult die Patienten im Selbstmanagement, führt diverse Untersuchungen und Tests durch, validiert die Dokumentation des Patienten, leitet ihn durch das Programm, ist verantwortlich für die komplette Dokumentation der Programmelemente und unterstützt den Arzt, dass regelmässig alle vorgesehenen Untersuchungen durchgeführt werden, zum Beispiel eine Untersuchung der Augen. "Wichtig ist die Abgrenzung zum Arzt», sagte Joos. «Bei uns ist genau aufgeschrieben, was ich darf und was nicht, wann ich den Arzt dazu holen muss und wann es ein Notfall ist.» Am Anfang seien die Ärzte skeptisch gewesen, erzählt sie. «Aber als positive Rückmeldungen von den Patienten kamen, schickten sie gleich die nächsten zu mir.» Das häufigste CCM-Programm, Hypertonie und Diabetes Typ 2, erfülle die Kriterien eines guten DMP, berichtete Jungi. Im Schnitt haben 68 Prozent der Patienten ihr jeweiliges Ziel erreicht, also etwa den Ziel-Blutdruck oder eine Änderung des Lebensstils. CCM im Hausarztbereich lasse sich implementieren, so das Fazit des Allgemeininternisten, und für alle Beteiligten praktizierbar. "Wer gute Ideen hat, sollte sich nicht durch noch nicht ausgereifte Rahmenbedingungen abschrecken lassen", schloss Jungi. Interprofessionelles Arbeiten sei wichtig, «und ausserdem macht es mehr Spass».

\section{Bildnachweis}

Felicitas Witte

Bei Sanacare gibt es fünf CCM-Programme: 1 . Diabetes Typ 2, 2. arterielle Hypertonie, 3. COPD, 4. Hypertonie und Diabetes, 5. Hypertonie und COPD. Am CCM spielen verschiedene Akteure mit. Ein Behandlungsteam

\footnotetext{
Literatur

1 Starfield B, Shi L. The medical home, access to care, and insurance: a review of evidence. Pediatrics 2004;113:1493-8.
} 